\section{Readers' thoughts on treating low back pain with counterstrain technique}

\section{To the Editor:}

This is in response to the article by Maurice Ramirez and colleagues, "Low back pain: Diagnosis by six newly discovered sacral tender points and treatment with counterstrain technique" (JAOA 1989;89:905-913). I wish to commend the authors for their astute observations, including the previously unreported tender points on the posterior aspect of the sacrum. I would like to offer another interpretation of their definition of "flexion of the sacrum."

Motion of any part of the body must be related to some other reference point, such as an adjacent part, or the "center of gravity," located within the pelvic girdle anterior to the first sacral segment. Specifically, motions of the sacrum traditionally have been related to the iliac portion of the innominate bones.

The standard definition of "flexion of the sacrum" is an anteriorinferior movement of the base of the sacrum in relation to the ilia to which it articulates bilaterally. The posterior movement of the apex of the sacrum and coccyx away from the symphysis pubis should be included in this definition as well.

Kapandji ${ }^{1}$ describes sacral motion similarly to the aforementioned definition. However, he also includes the motion of the pelvic bones among themselves as well as their relationship to the sacrum.
The plane of the articular surface on the lateral aspect of the sacrum at its junction with the ilia diverges anteriorly. This means that the sacrum is wider anteriorly but narrower posteriorly. The ilia rotate around the sacrum on this divergent plane.

As such, the distance between the iliac crests is shorter, whereby they move medially closer to the midline of the pelvis. The degree of movement here ranges between $3 \mathrm{~mm}$ and $13 \mathrm{~mm}$. At the same time, the ischial tuberositates move laterally, between $15 \mathrm{~mm}$ and 17.5 $\mathrm{mm}$.

Thus, depending on the flexion or extension of the sacrum (which Kapandji calls nutation or counternutation, respectively), the diameter change of the pelvic inlet and outlet will influence the progress of labor during childbirth.

With a woman in labor, lying in the supine position with the weight on the apex of the sacrum (moving the base posteriorly) and legs extended, the thigh flexor muscles rotate the ilia anteriorly on the sacrum into sacral extension (counternutation). The pelvic inlet widens, facilitating descent of the fetal head. Thighs flexed on the abdomen cause the hamstring muscles to rotate the ilia posteriorly on the sacrum into sacral flexion (nutation). The pelvic outlet widens for the final stage of labor and delivery.

Kapandji also discusses the rotation axis of the sacrum between the ilia. However, he mentions only a horizontal transverse axis, which according to his research, has been located at different sites. Yet, the classic axis is located in the posterior sacroiliac ligaments as well as the ligament band extending from the posterior iliac crest to the tubercle of the second sacral segment.

Other investigators place the axis within the sacroiliac joint at the junction of the cephalic and caudal portions of this auricularshaped joint. Still other investigators place the axis several centimeters anterior to the second sacral segment.

It appears, then, that more than a single transverse axis exists, around which the sacrum moves between the ilia. This depends on whether the patient stands erect or bends forward from the hips with knees extended; or is in the supine or prone position.

Further complicating matters, the involuntary motion of the sacrum must be considered. This motion is synchronized with motion at the sphenobasilar symphysis in the base of the cranium. In my opinion, the axis of rotation for the involuntary motion of the sacrum between the ilia is $10 \mathrm{~cm}$ to $20 \mathrm{~cm}$ posterior to the second sacral segment.

In my research, I discovered that when a standing person bends completely forward from the hips with knees extended, the sacrum is in the posterior position and the iliac crests are separated as much as $4 \mathrm{~cm}$ to $8 \mathrm{~cm}$. (These measurements were recorded using double-exposed $\mathrm{x}$ ray films.)

Flexion of the vertebral column is defined as forward bending. This motion approximates the anterior borders of all vertebrae, including the fifth lumbar on the sacrum. In this forward flexion position, the base of the (continued on page 1384) 


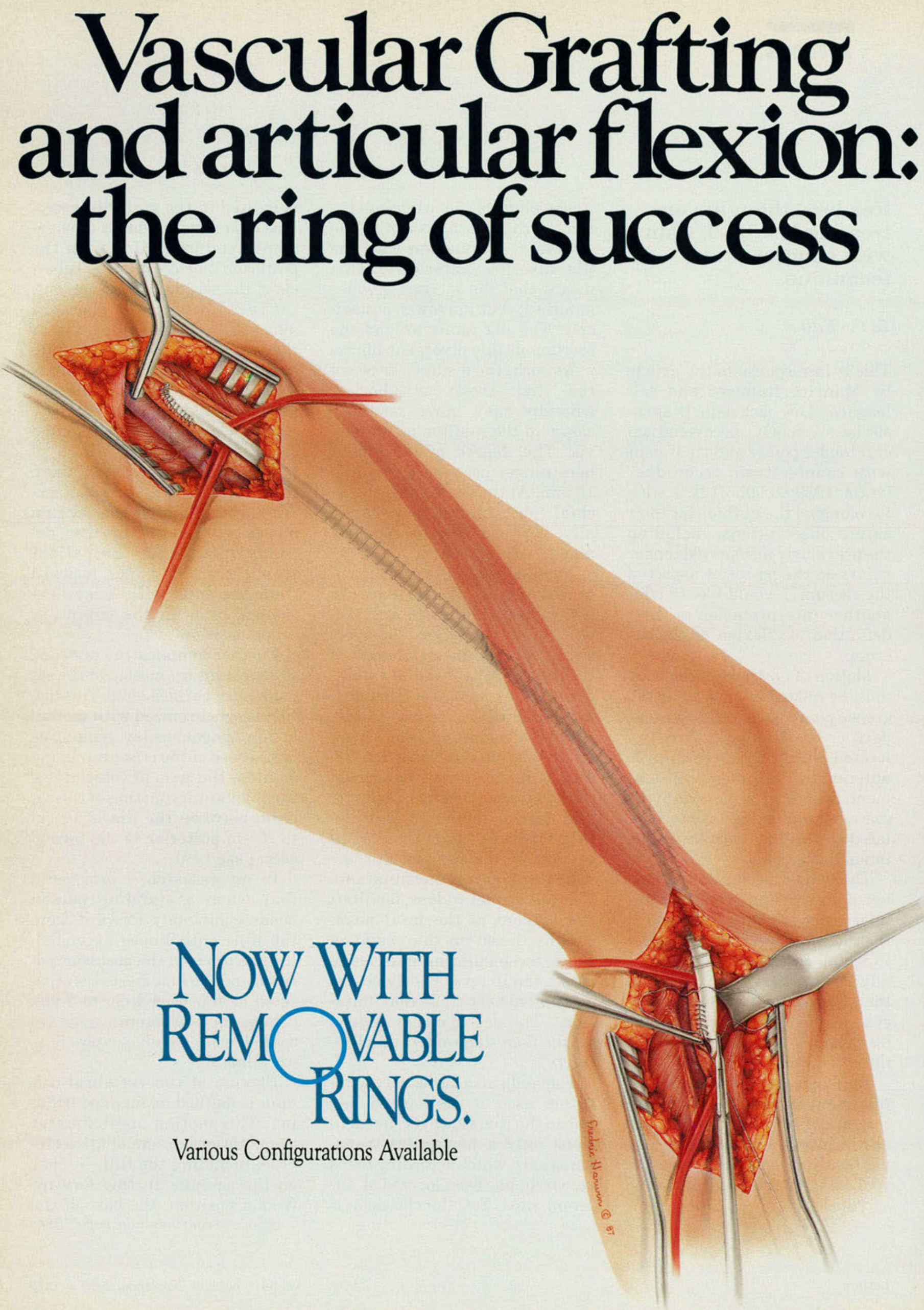


Standing, sitting, kneeling.... vascular graft crossing a point of articulation is exposed to the full range of motion inherent in the joint. Where the potential of compression of a prosthesis exists, the FEP RINGED GORE-TEX ${ }^{\circledR}$ VASCULAR GRAFT resists external pressures.

GORE-TEX Vascular Grafts may relieve lower limb ischemia with belowknee femoropopliteal bypass in the patient whose own veins cannot be used. Where the surgeon is concerned with joint flexion or other compressive forces, the external support provided by the

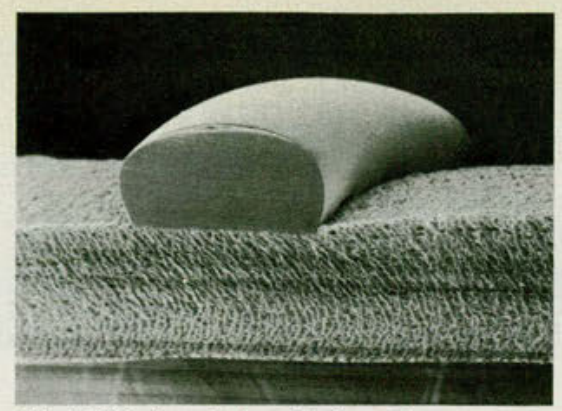

The FEP ring is bonded to the outer wall of the graft without compromising wall integrity, thickness or internal diameter. FEP (fluorinated ethylene propylene) is in the same polymer family as PTFE and exhibits equivalent inertness and biocompatibility. $(30 x)$

FEP RINGED GORE-TEX VASCULAR GRAFT is the natural choice.

The rings encircle the same Standard or ThinWalled GORE-TEX Vascular Graft whose handling and performance surgeons have come to know through more than 15 years and a million implants.

The GORE-TEX ${ }^{\mathrm{TM}}$ Suture offers specific advantages when sewing the FEP Ringed GORE-TEX Vascular Graft to the artery. The absence of plastic memory in this smooth, pliant material provides superior handling and easier, faster knot placement. Our needles are strong enough for the most demanding anastomoses and GORE-TEX Sutures are available in a wide variety of sizes.

For more information please use coupon below or call toll free, 800-638-4804.

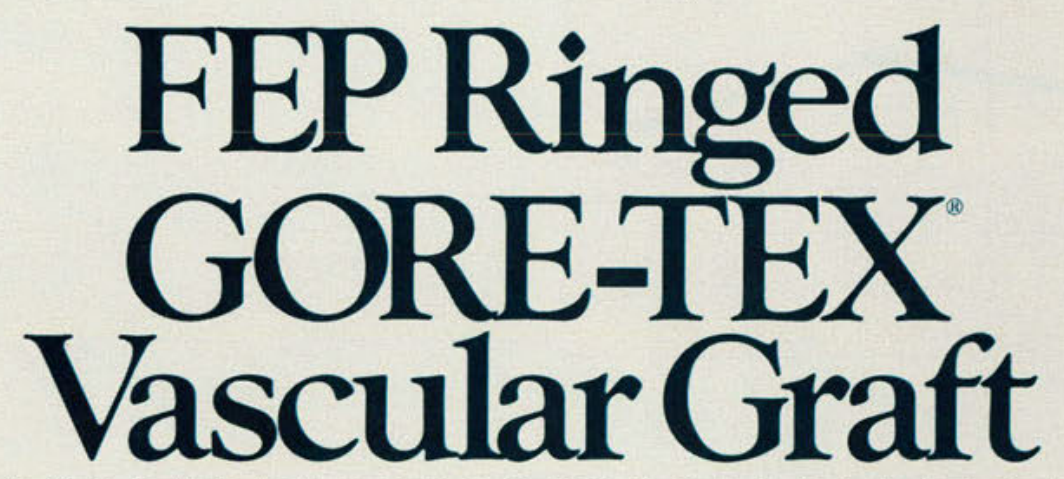

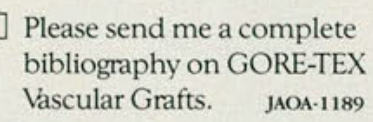

Please send me a complete bibliography on GORE-TEX Vascular Grafts. JAOA-1189

Name

\section{Address}

\begin{tabular}{lll}
\hline City & State & Zip \\
\hline Surgical Specialty & Phone \\
\hline Hospital Affiliation & \\
\hline W. Gore \& Associates, Inc, Post Office Box 1220, Route 213 North, Elkton, MD 21921
\end{tabular}

Please have your representative call for an appointment.

$\square$ Please send me information on the GORE-TEX Suture.

W.L. Gore \& Associates, Inc., Post Office Box 1220, Route 213 North, Elkton, MD 21921 


\section{Ansaid $100_{\text {mo: }} 1$ Tablet BID or TID}

\section{for Arthritis}

\section{Osteoarthritis}

ANSAID Tablets lessen disability

- Diagnosis: Osteoarthritis of the knee.

- Study: Double blind, randomized.

- Treatment: Ansaid, up to $200 \mathrm{mg} / \mathrm{day}$, or aspirin, up to $4,000 \mathrm{mg} /$ day.*

\section{Percent patients improved}

Investigators' assessment

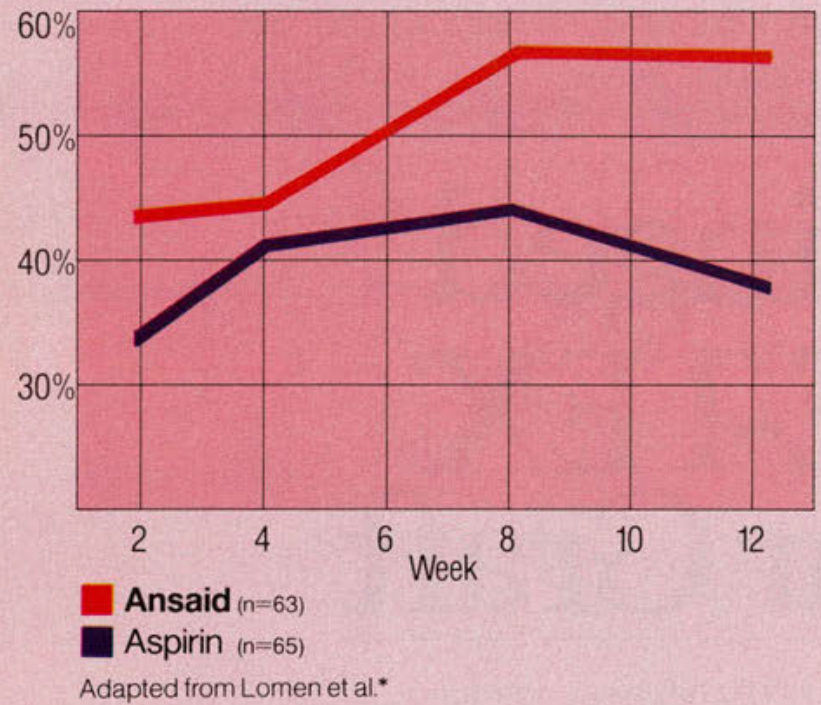

\section{Rheumatoid arthritis}

ANSAID Tablets improve mobility

A 52-week, double-blind, randomized study of 822 patients in the United States demonstrated that Ansaid (flurbiprofen) 200 to $300 \mathrm{mg} /$ day relieves rheumatoid arthritis pain and inflammation as effectively as comparable dosages of aspirin.

Efficacy was confirmed by improvement in such parameters as grip strength, duration of morning stiffness, and time to onset of fatigue

\section{Effective...improves patient mobility}




\section{EFFICACY SAFETY EXPERIENCE}

\section{An excellent}

\section{safety record worldwide}

- 1 billion patient days of treatment with flurbiprofen since 1977.

- Experience in 70 countries.

Incidence of side effects in three body systems during clinical trials with ANSAID

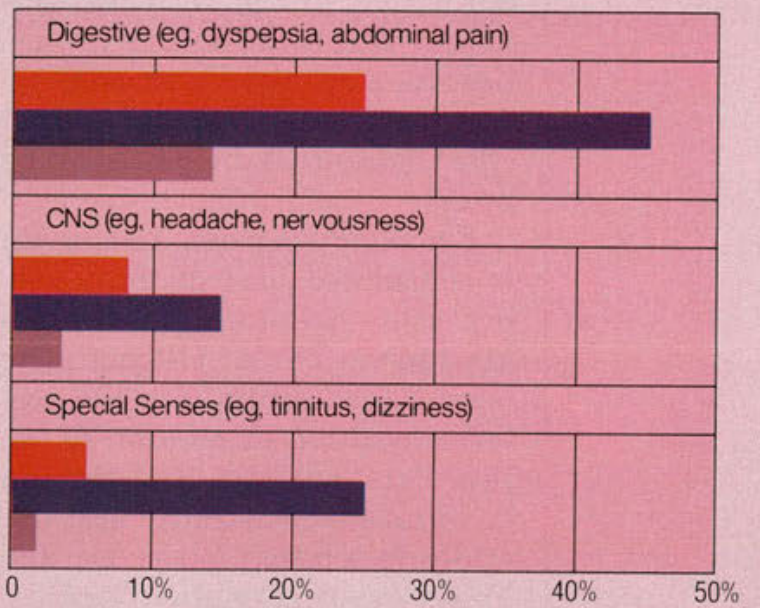

Ansaid $(n=2.820)$

Aspirin $(n=626)$

Placebo $(n=274)$

\section{Upjohn}

\section{The Upjohn Company}

Kalamazoo, MI 49001, USA

\section{Convenient dosage}

The dosage of Ansaid tablets

is $200 \mathrm{mg}$ or $300 \mathrm{mg}$ daily, administered

BID or TID. (Most experience in

RA has been with TID or QID dosage.)

Recommended starting dosage is $100 \mathrm{mg}$ BID.

\section{*Lomen PL, Lamborn KR, Porter GH, et al.}

Treatment of osteoarthritis of the knee: A comparison of flurbiprofen and aspirin. Am JMed. 1986;80 (suppl 3A):97-102.

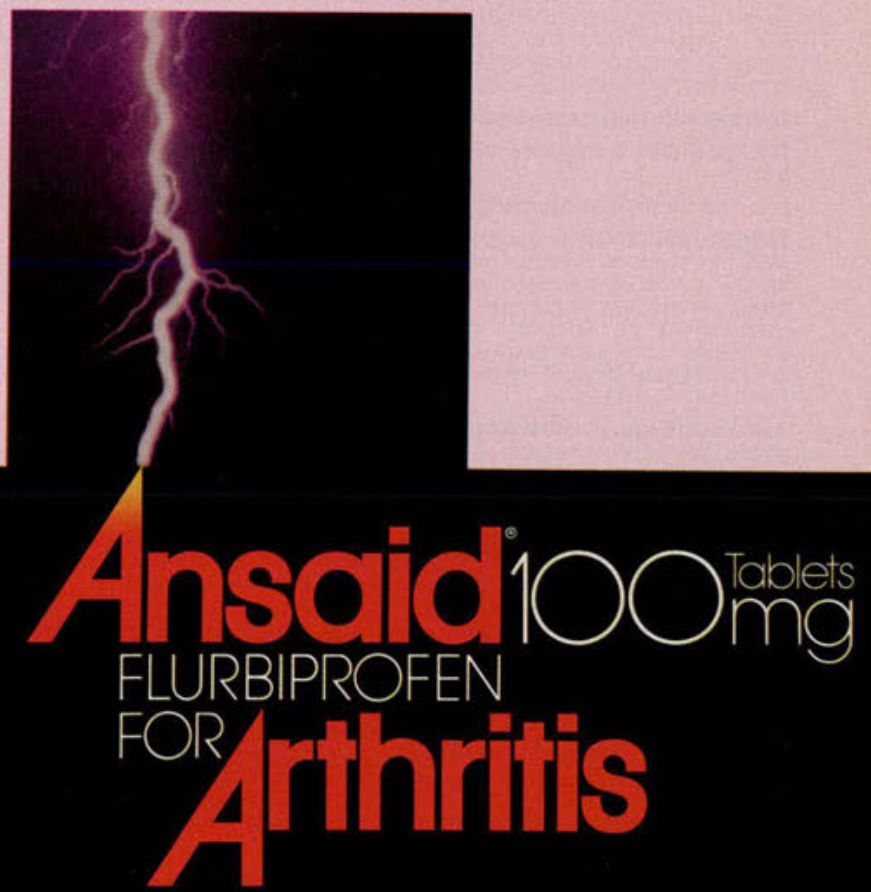




\section{Ansaidiooma \\ letters \\ (continued)}

INDICATIONS: Acute and long term treatment of signs and symptoms of rheumatoid arthritis and osteoarthritis.

CONTRAINDICATIONS: Hypersensitivity to ANSAID, or if aspirin or any other nonsteroidal antiinflammatory agent induces asthma, urticaria or other allergic type reactions. Fatal asthmatic reactions have been reported in such patients.

WARNINGS: Gastrointestinal effects: Risk of Gl ulcerations, bleeding and perforation with nonstero dal anti-inflammatory therapy: Serious GI toxicity can occur at any time, with or without warning symptoms, during chronic treatment. The occurrence is about $1 \%$ after $3-6$ months, $2-4 \%$ after a year. Patients should be informed of signs and symptoms of serious Gl toxicity and what to do if it occurs. No subset of patients not at risk has been identified. Prior history of serious Gl events and other risk factors o peptic ulcer disease, e.g. alcoholism, smoking, etc., have been associated with increased risk. The elderly and debilitated tolerate ulceration and bleeding less well. Higher doses probably carry a greater risk Gl ulceration and bleeding can occur without warning symptoms and chronically treated patients should be followed.

PRECAUTIONS: Patients with impaired renal or hepatic function: Use ANSAID and similar agents cautiously. Pharmacokinetics have not been studied in patients with decreased liver function.

Renal Effects: Rats develop renal papillary necrosis at dosages equivalent to human therapeutic levels, as do monkeys given 20-40 times the human dose. In clinical studies of ANSAID, kidney function tests were done monthly and renal effects were similar to those seen with other nonsteroidal anti-inflammatory drugs. A second form of renal toxicity has been seen in patients with prerenal conditions that reduce rena blood flow or blood volume. A nonsteroidal anti-inflammatory drug may cause dose-dependent reduction in prostaglandin formation and precipitate overt renal decompensation. Patients at greatest risk are those with impaired renal or hepatic function, heart failure, those taking diuretics or the elderly. Drug discontinuation usually leads to recovery. Patients at high risk on chronic treatment should have rena function monitored if they have signs or symptoms that may be consistent with mild azotemia, eg malaise, or symptom flubs apretice or symptoms. Fl resto avoid accumulation of flurbiprofen metabolites.

Liver tests: Borderline elevations of liver function tests may occur in up to $15 \%$ of patients, and may progress, remain unchanged or disappear with continued treatment. Patients with signs and/or symptoms or with an abnormal liver function test should be evaluated further.

Anemia: Patients treated long term who have initial hemoglobin values under $10 \mathrm{~g} / \mathrm{dL}$, should have periodic hemoglobin values.

Fluid retention and edema: Fluid retention and edema have been reported so use ANSAID with caution in patients with conditions such as cardiac decompensation or hypertension.

Vision Changes: Blurred and/or diminished vision has been reported. Patients with eye complaints should have periodic ophthalmologic exams.

Effect on platelets and coagulation: Platelet aggregation is inhibited and bleeding time prolonged, patients who may be adversely affected should be carefully observed.

Information for patients: Physicians and patients may wish to discuss potential risks and likely benefits.

Drug Interactions: Anticoagulants: Bleeding parameters are affected, clinical bleeding has been reported. Aspirin: Flurbiprofen levels were $50 \%$ lower. Concurrent use is not recommended. Betaadrenergic Blockers: Pharmacokinetics and heart rate reduction are not affected, hypotensive adrenergic Blockers. Pharmacokinetics and heart rate reduction are not affected, hypotensive increase in area under the flurbiprofen serum concentration curve. Diuretics: Patients receiving increase in area under the flurbiprofen serum concentration curve. Diuretics: Patients
furosemide or thiazides should be closely observed to make sure the desired effect is obtained.

Carcinogenesis, mutagenesis, impairment of fertility: № evidence.

Teratogenic effects: Pregnancy category B: No effect in animals. Not recommended for use in pregnancy.

Labor and delivery, nursing mothers, pediatric use: Use is not recommended.

ADVERSE REACTIONS: $9.4 \%$ of 4123 patients dropped out of studies because of an a.dr. Incidence >1\%: Gastrointestinal: Dyspepsia*, diarrhea*, abdominal pain*, nausea*, constipation, Gl bleeding flatulence, elevated liver enzymes and vomiting 'Central nervous system: Headache*, "stimulation" (e. anxiety, insomnia, reflexes increased, tremor) and "inhibition" (e.g. amnesia, asthenia, somnolence, malaise and depression). Respiratory: Rhinitis. Dermatologic: Rash. Special senses: Dizziness, tinnitus and changes in vision. Genitourinary: Signs and symptoms suggesting a urinary tract infection* Body as a whole: Edema* Metabolic/nutritional: Body weight changes

* Reaction in 3 to $7 \%$ of patients.

Incidence <1\% (Causal relationship probable): Gastrointestinal: Peptic ulcer disease (See Warnings) gastritis, bloody diarrhea stomatitis, esophageal disease, hematemesis and hepatitis, cholestatic and non-cholestatic jaundice. Central nervous system: Ataxia, cerebrovascular ischemia, confusion paresthesia and twitching Hematologic: Decrease in hemoglobin and hematocrit, iron deficiency anemia, leukopenia, eosinophilia and ecchymosis, thrombocytopenia, hemolytic anemia and aplastic anemia. (See Precautions) Respiratory: Asthma and epistaxis. Dermatologic: Angioedema, urticaria, eczema and pruritus; photosensitivity, toxic epidermal necrolysis and exfoliative dermatitis. Specia senses: Conjunctivitis and parosmia. Genitourinary: Hematuria and impairment of renal function interstitial nephritis. Body as a whole: Anaphylactic reactions, chills, fever. Metabolic/Nutritiona: Hyperuricemia. Cardiovascular: Heart failure, hypertension, vascular disease and vasodilatation.

Incidence <1\% (Causal relationship unknown): Gastrointestinal: Periodontal abscess, appetite changes, cholecystitis and dry mouth. CNS: Convulsion, meningitis, hypertonia, cerebrovascular accident, emotional lability and subarachnoid hemorrhage. Hematologic: Lymphadenopathy. Respiratory: Bronchitis, laryngitis, dyspnea, pulmonary embolism, pulmonary infarct, hyperventilation. Dermatologic: Alopecia, nail disorder, herpes, dry skin and sweating. Special senses: Ear disease, corneal opacity glaucoma retrobulbar neuritis, change in taste transient hearing loss, retinal hemorrhare Genitourinary: Menstrual disturbances, vaginal and uterine hemorrhage vulvovaginitis, prostate disease. Metabolic/nutritional: Hyperkalemia. Cardiovascular: Arrhythmias, angina pectoris and myocardial intarction. Musculoskeletal: Myasthenia.

DOSAGE AND ADMINISTRATION: 200 to $300 \mathrm{mg}$ daily, administered bid, tid or qid. (Most experience in rheumatoid arthritis has been with tid or qid dosage). Dose should be tailored to severity of symptoms and patient response.

Store at controlled room temperature $\left(15-30^{\circ} \mathrm{C}\right)$.

Federal law prohibits dispensing without a prescription sacrum is in the posterior position between the ilia.

In more than 50 years as an osteopathic physician, I have recognized that the base of the sacrum moves anteriorly between the ilia when a person bends backward and that the base of the sacrum moves posteriorly between the ilia when a person bends forward. These motions occur whether a person stands or sits.

Thus, it appears the sacrum may be a transitional bone, depending on whether one considers it part of the vertebral column or part of the lower extremities. As such, descriptions of sacral motion as it relates to specific adjacent landmarks must be precise.

For example, when the sacrum is considered part of the vertebral column, the anterior borders are approximated in flexion. The base of the sacrum is in the posterior position in relation to the ilia. This indicates that the ilia have rotated anteriorly and laterally in relation to the sacrum. Thus, "external rotation" best describes this iliac position.

This term was first used by William Garner Sutherland, DO. Dr Sutherland, who discovered the cranial concept, used "external rotation" to describe all paired bones during the flexion of the midline bones in the craniosacral mechanism. It is now accepted that all paired bones of the cranium move away from the midline into external rotation during the flexion of the sphenobasilar symphysis. Thus the transverse skull diameter widens. Simultaneously, the pull of the spinal dura mater at its attachment to the second sacral segment (continued on page 1387) 


\section{Coming in ...}

\section{THE DO}

DOs can stop losing sleep over their patients' nocturnal illnesses-The December issue of The DO focuses on sleep disorders and research. The issue will include the latest information on assessing and treating common sleep disorders as well as a survey of current sleep research efforts.

Also in the December issue: the availability of new cancer drugs and a look at how Washington state's innovative insurance plan is faring.

\section{JAOA}

- Endoprosthesis insertion for malignant obstructive jaundice: A retrospective review

- AIDS-associated malignancies

- HIV disease training: A 'how-to' guide

- Transhiatal blunt esophagectomy for carcinoma of the esophagus

- The radiology of stress fractures

- Myocarditis in the 1980s: A clinical perspective

- The use of platelets in transfusion medicine

- War, politics, and osteopathic medicine

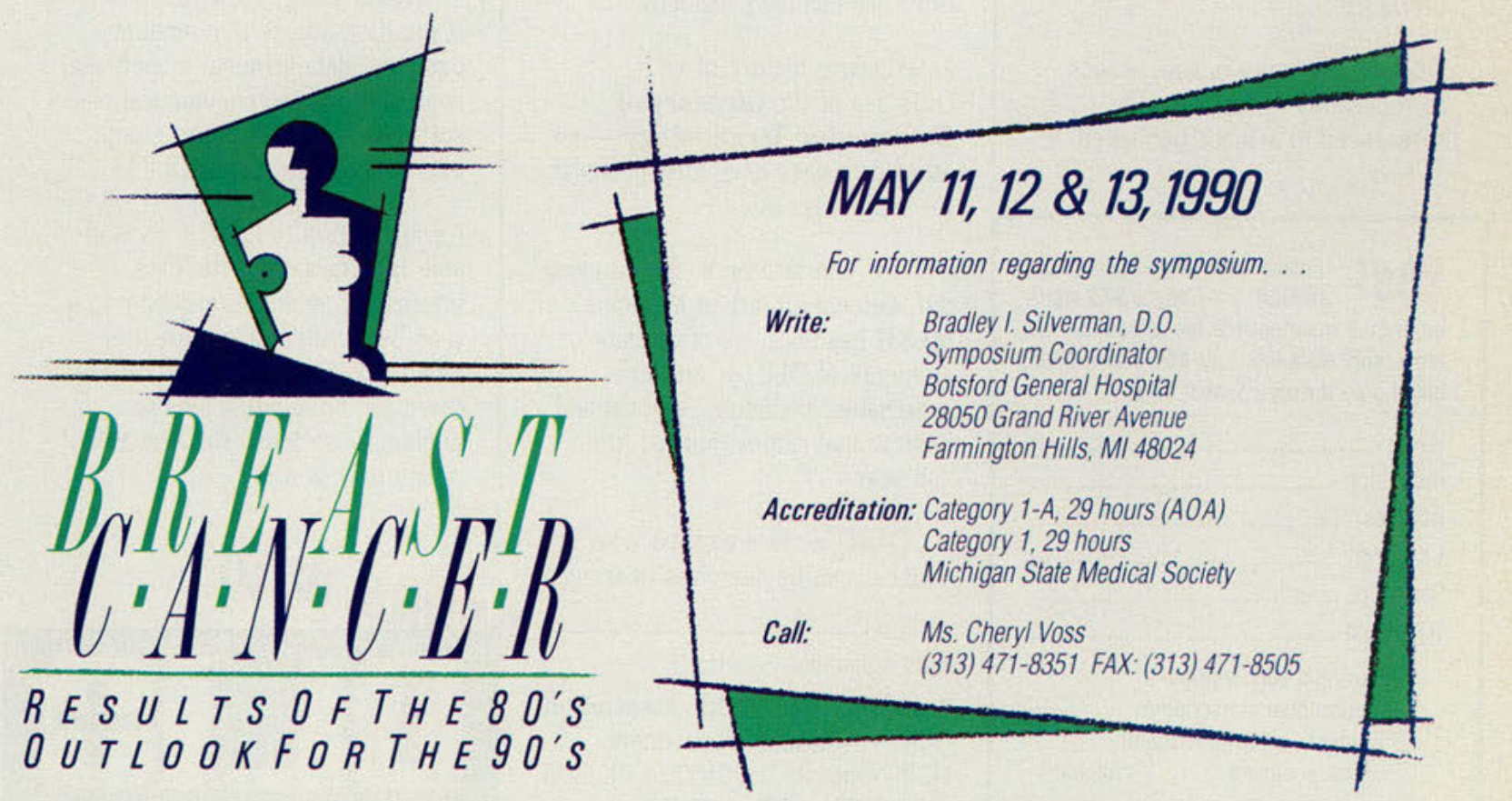




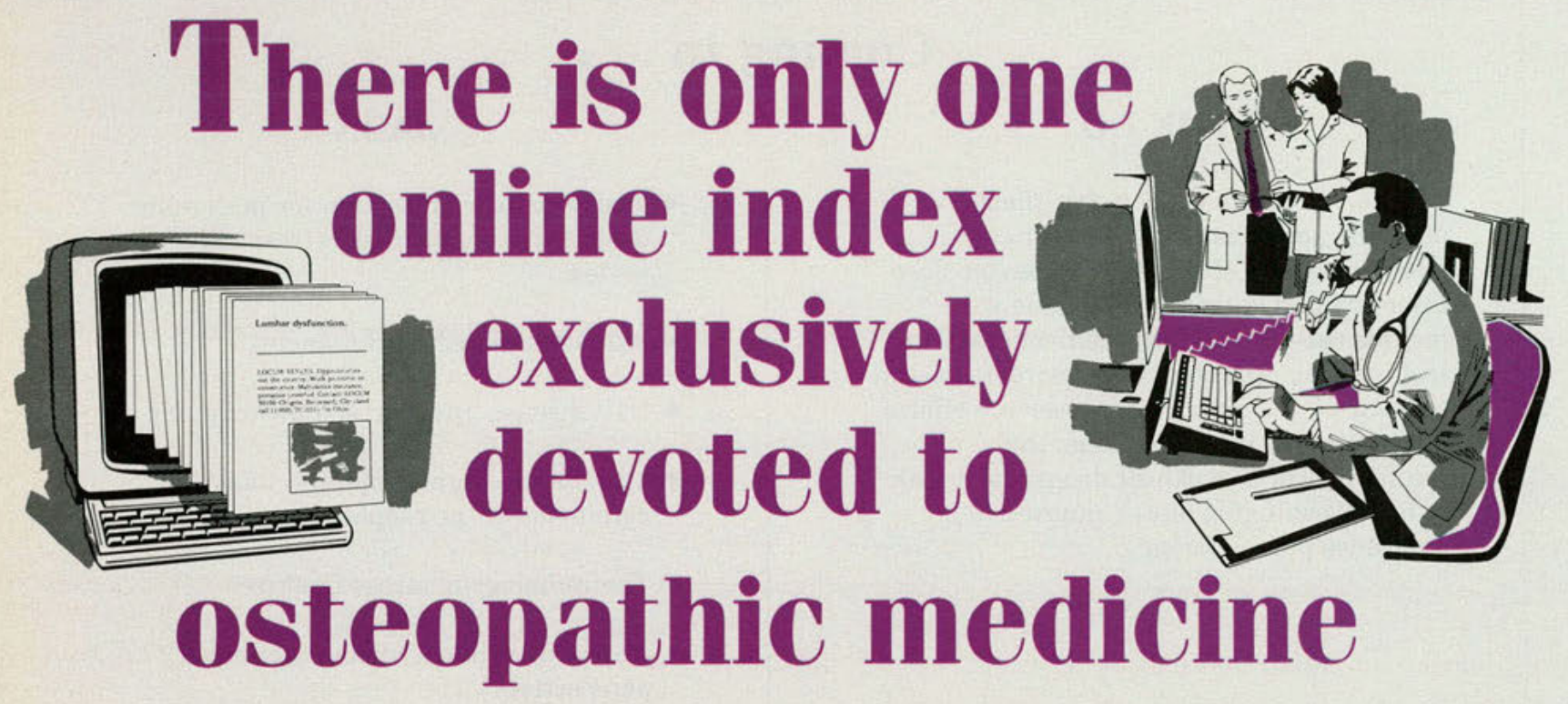

That index is $\mathrm{OLIO}+\ldots$ the Osteopathic Literature Index/Online.

OLIO + is unique. OLIO + is the only bibliographic data base that offers comprehensive coverage of the osteopathic medical literature.

$\mathrm{OLIO}+$ subscribers have access to thousands of bibliographic references to articles published

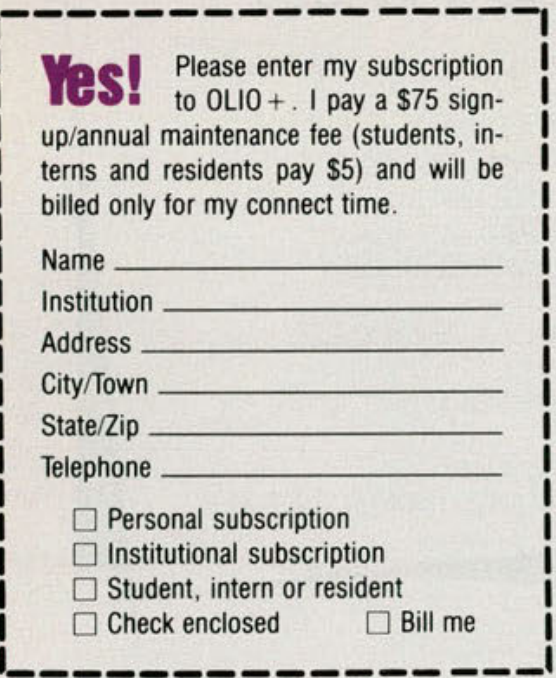

since the mid-1970s in major osteopathic journals. Clinical articles, convention reports, legislative and economic information, and news and feature articles can all be located quickly and easily with $\mathrm{OLIO}+$. New records are added every month, and new journal titles are included regularly.

An exclusive feature of $\mathrm{OLIO}+$ is its use of the Glossary of Osteopathic Terminology - no other data base uses this vocabulary for subject access.

$\mathrm{OLIO}+$ can also be searched using the National Library of Medicine's MeSH headings. All other data elements of $\mathrm{OLIO}+$ are also searchable, including personal and institutional names, journal title and year.

OLIO + is easy to use. $\mathrm{OLIO}+$ can be searched by using a

Send registration form to:

\section{American 0steopathic Association} OLIO + /Editorial Department 142 E. Ontario St., Chicago, IL 60611 $800 / 621-1773 \times 5865$ or 5895 common command language or a search menu. References contain complete bibliographic information and can be displayed in one of three formats. Searches can be stored for later reference or downloaded to your personal computer.

OLIO + is inexpensive. If you have access to a modemequipped data terminal or personal computer and telecommunications software, you have all the equipment needed to access OLIO + .

A subscription to OLIO + is available for $\$ 75$ a year; students, interns and residents pay only $\$ 5$ a year. Subscribers are thereafter billed only for their connect time; there are $n o$ monthly fees or minimum-use fees. The first $\$ 60$ of online time is free.

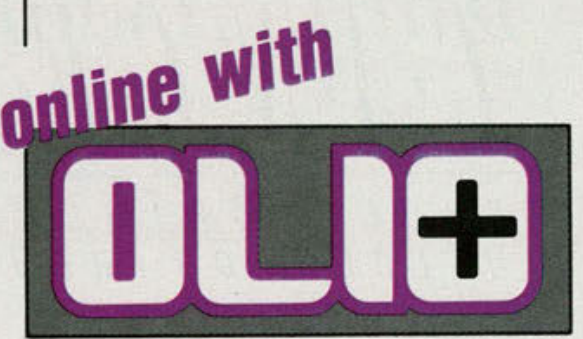


within the sacral canal lifts the base of the sacrum into the posterior position between the ilia. At the same time, the ilia move anteriorly and laterally into the position of external rotation. This movement causes the pelvic inlet to widen.

Finally, no discussion of this subject would be complete without mentioning locomotion. During walking, the pelvis and sacrum move around the two oblique axes. $^{2}$

THOMAS F. SCHOOLEY, DO Knoxville, Tenn

1. Kapandji, IA: The Physiology of Joints, ed 2. New York, Churchill-Livingstone, 1974, vol

2. Schooley TF:Osteopathic Principles and Practice. Newark, Ohio, American Academy of Osteopathy, to be published.

\section{Response:}

Thank you for your interest in our work in the area of manipulation. The original draft of our article used the terms "nutation" and "counternutation,"referring to the works of Drs Viola Frymann and Gerald Cooper. As a member of the Cranial Academy, I personally prefer the use of these terms as opposed to the physiologically incorrect definition of sacral flexion and extension, which is based on a whole body reference system. The latter was used in the article to prevent confusion among readers who are not familiar with the terms "nutation" and "counternutation."

\section{MAURICE A. RAMIREZ}

University of Osteopathic

Medicine and Health Sciences
College of Osteopathic Medicine and Surgery

Des Moines, Ia

\section{To the Editor:}

Regarding the article by Ramirez and colleagues (JAOA 1989; 89:905-913), the documentation of the previously undescribed medial sacral tender points is a welcome addition to counterstrain and to the often frustrating field of low back pain management. However, I found the approach to treatment of the medial sacral tender points too fatiguing for operators who are short in stature. Therefore, I would like to offer an alternative approach.

I treat the medial sacral tender points and all lumbar and sacroiliac and trochanteric tender points by placing a pillow under the thigh and knee of the prone patient, inducing extension of the lumbar spine, sacrum, and hip. Having achieved some degree of extension with the pillow under the thigh on the affected side, the physician stands on the same side as the tenderpoint with a finger of the superior hand monitoring the tender point.

With the inferior hand under the patient's ankle, flex the lower leg on the patient's knee to a 90 degree angle. Depending on the tender point to be treated (sacral, lumbar, or sacroiliac), the knee may be flexed or extended 45 degrees from this starting position. Varying amounts of adduction or abduction and internal or external rotation of the foot or thigh may be required to fine tune the position for a given tender point.

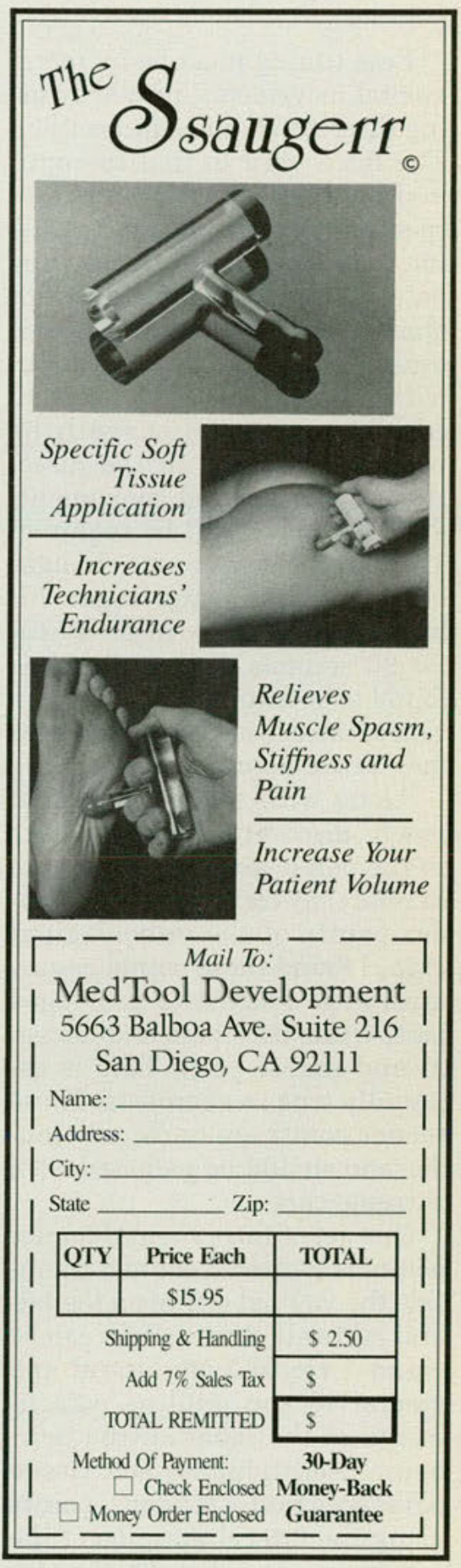


Fine tuning must be by incremental movements, primarily using discrete changes in position. The force used to find the optimal position should be the least required to overcome inertia, using deep, slow respirations by the patient, gravity and the inherent elastic recoil of the antagonistic muscles. If the operator works with his or her eyes closed, and coordinates respirations with the patient, "looking" in the direction of the desired movement, even less force will be required. The perception of tissue changes under the monitoring finger will be enhanced. The position is held for 90 seconds. If the release is found to be incomplete, introduction of muscle energy techniques may prove appropriate.

Along with this discussion of newly discovered tender points in the pelvic girdle, I would like to relate my experience with tender points of the vulvovaginal area. I found these points associated with delayed or prolonged postpartum back pain and coccygal and sacral pain. This is especially true in gymnasts. These tender points are unusually tender and should be palpated with extreme care.

One set of intravaginal tender points is located $2 \mathrm{~cm}$ to $3 \mathrm{~cm}$ inside the vaginal orifice on the lateral midwall. A second set can be found $1 \mathrm{~cm}$ to $2 \mathrm{~cm}$ lateral and medial to the midline attachments of the labia on the perineum. Additionally, soft tissue paracoccygeal tender points along the lateral margins of the coccyx may be noted. This tender point grouping suggests somatic dysfunction in the pubococcygeal ligaments. Subjective symptoms include abdominal pain, vague perineal distress, and referred pain to the coccygeal and low back regions.

My first experience with this particular pattern was with a young adult acrobatic dancer. She had undergone a barium enema, CT scan, intravenous pyelogram (IVP), and mental health evaluation for accusations of malingering. The patient had been taking multiple antibiotic regimens for pelvic inflammatory disease.

After the patient failed to improve after manipulation directed at the low back and sacral tender points, treatment was directed at the pelvic floor. Eradication of the vaginal wall and coccygeal tender points resulted in almost immediate symptom relief, which occurred within two treatments.

Treating the first set of intravaginal tender points requires that the patient lie supine with the legs flexed at the hips and the ankles crossed. The physician's foot is placed on the table with the knee flexed 90 degrees. The physician's gloved finger monitors the tender point intravaginally, while the opposite hand supports the patient's crossed ankles, which rest on the physician's knee. The patient's flexed knees are abducted away from each other while the operator moves the crossed ankles toward or across the midline.

The second set of tender points is manipulated in the following manner: With the patient's hips flexed 90 degress and ankles placed on the physician's knee as previously described, the patient's ipsilateral knee is ab- ducted and the contralateral knee is kept in the midline. At the same time, the operator adjusts the degree of flexion at the hips as indicated by the tender point under the monitoring finger.

Alternatively, either set of tender points may be treated with the patient supine, hips flexed 90 degrees, ankles on the physician's inferior knee, and slight abduction of the knees while the operator rotates the pelvis by adducting the lower legs toward the midline. Fine tuning of this third position may be performed by internal rotation and inversion of the feet.

(These manipulative procedures are somewhat similar to Jones' description of treatment for Iliacus tender point dysfunction.)

As with most anterior lesions, some delay may occur in the onset of relaxation at the tender point. Once the physician locates the patient's ideal comfort position, the operator must be patient. It is important not to apply pressure to the tender point if a long delay ensues in obtaining the release of tension at the point. This will only prolong the wait. Furthermore, no pressure should be applied to the tender point during the 90 -second holding period.

The posterior coccygeal tender points are treated similarly to those previously described procedures for lumbar and sacral lesions.

Short-statured operators may be more comfortable performing these maneuvers from a low foot stool. As in all counterstrain procedures, movements should be (continued on page 1394) 


\section{THE EMERGENCE OFA NEW SOURCE OF 24-HOUR ANTIHYPERTENSIVE AND ANTIANGINAL PROTECTION}

New, Once-a-Day

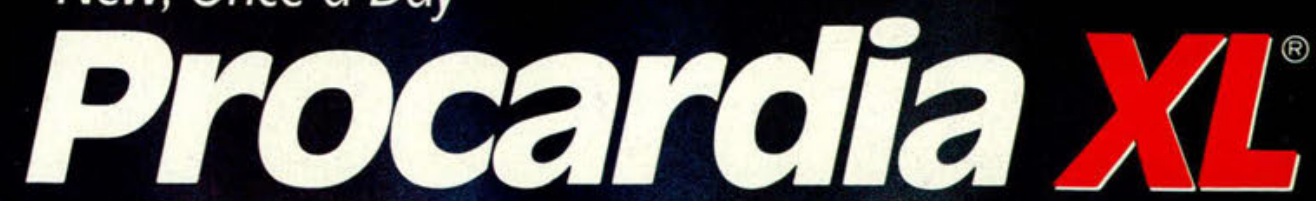

(nifedipine) Extended Release

Tablets $30 \mathrm{mg}, 60 \mathrm{mg}$ and $90 \mathrm{mg}$ GITS 


\section{Once-a-Day Procarala XI (nifedipine) Extended Release Tablets $30 \mathrm{mg}, 60 \mathrm{mg}$ and $90 \mathrm{mg}$ GITS 24-HOUR CONIROL}

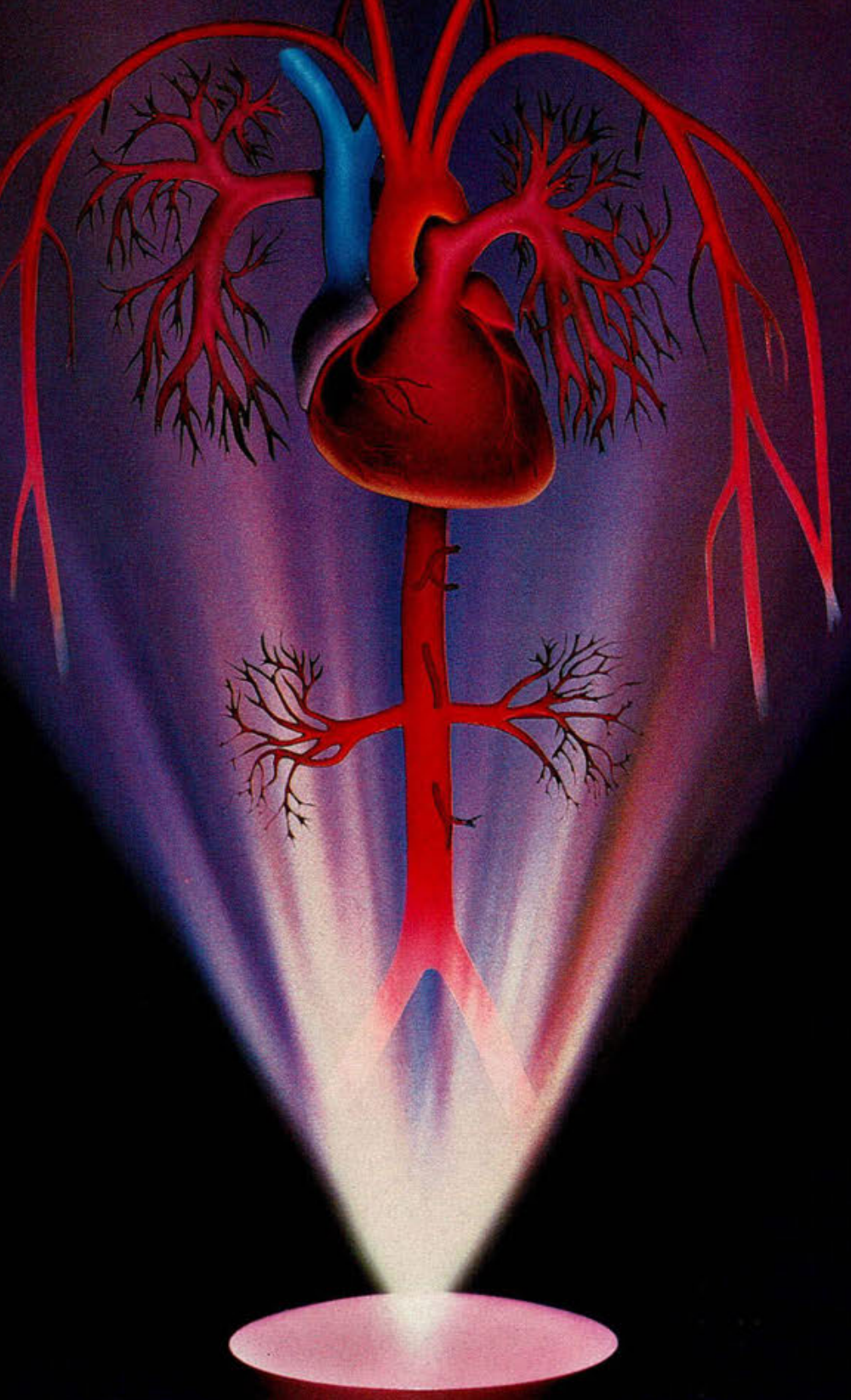




\section{FOR BOIH HYPERTENSION AND ANGINA WITH ONCE-DALLY DOSING}

\section{NOW, Once-Daily Dosing Controls Hypertension}

- The only calcium channel blocker indicated for once-a-day dosing at all doses

- Effective as monotherapy ${ }^{1}$ and in combination ${ }^{2}$

\section{Once-Daily Dosing Controls Angina}

- The only once-a-day calcium channel blocker for angina

- Easy to switch from nifedipine capsules to PROCARDIA XL Extended Release Tablets ${ }^{3.4}$

- PROCARDIA XL angina indications: Patients with proven or suspected vasospastic angina, and patients with classic effort angina who remain symptomatic despite adequate doses of beta blockers and/or nitrates or who cannot tolerate these agents

\section{New 24-Hour Controlled-Release Delivery System}

- Releases nifedipine into the gastrointestinal tract at an essentially constant rate over the 24-hour period, independent of $\mathrm{pH}$, with no dose dumping ${ }^{5.6}$

- Minimal serum fluctuations - no significant peaks, no significant troughs $^{5}$

- Favorable vasodilatory side-effects profile. The most common side effects are peripheral edema, which is not associated with fluid retention, and headache In controlled trials of 776 patients with PROCARDIA XL, edema resulted in discontinuation of therapy in $2.7 \%$ of patients. ${ }^{4}$ 
New, Once-a-Day

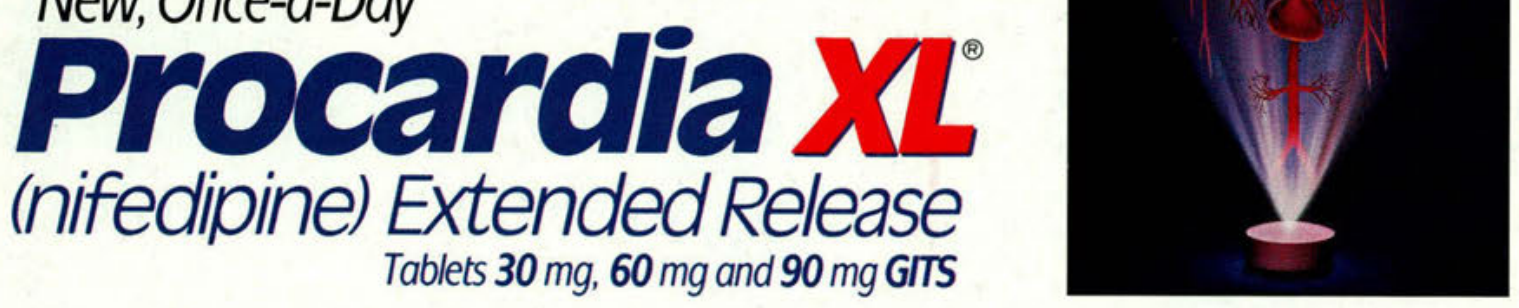

\section{4-HOUR CONTROL FOR BOTH HYPERTENSION AND ANGINA WITH ONCE-DAILY DOSING}

\section{EASY TO INITIATE ONCE-DAILY DOSING}

- Initiate once-a-day therapy with a single 30-mg or 60-mg PROCARDIA XL Extended Release Tablet, swallowed whole

\section{EASY TO SWITCH TO ONCE-DAILY DOSING}

- Over $90 \%$ of angina patients controlled on nifedipine capsules were easily switched and controlled on PROCARDIA XL Extended Release Tablets at the nearest total daily dose ${ }^{4}$

\section{TITRATION SHOULD PROCEED AS CLINICALLYWARRANTED}

- For full dosage instructions, see prescribing information

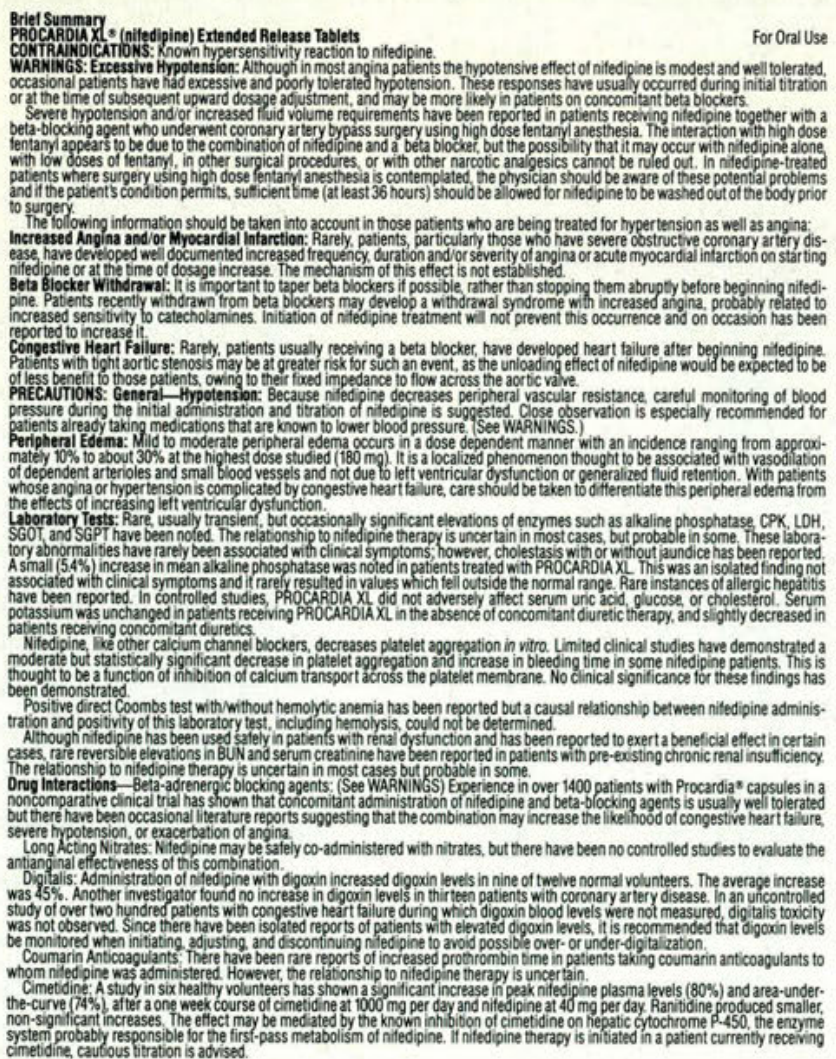

Carrinogenesis, Mutagenesis, Impairment of Fertility: Nitedipine was administered orally fo rats, for two years and was not shown to
be carcinogenic When given to rats prior to mating. nifedipine caused reduced tertility at a dose approximately 30 tmes the maximum

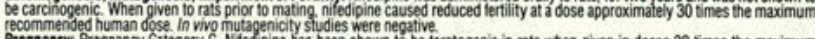

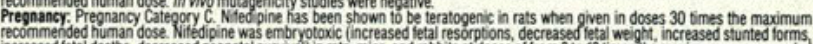
increased fetal deaths, decreased deonatal surviva) in rats, mice and rabbits at doses of from 3 to 10 times the maximum recommended

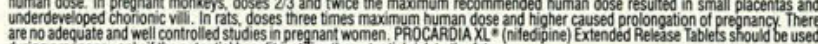

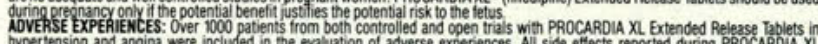
hypertension and angina were included in the evaluation of adverse experiences. All side effects reported during PROCARDIA XL reported with PROCARDOAXL Was ederma which was dose related and ranged in frequency from approximately $10 \%$ to about $30 \%$ at the

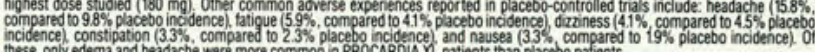
these only edema and headache were more common in PROCARD IA X Ratients than placeboo patients

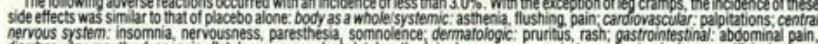

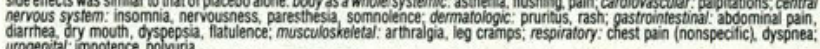
other adverse reaccions were reported sporadically with an incidence of $1.0 \%$ or less. These include: body as a whaleersystemic: face

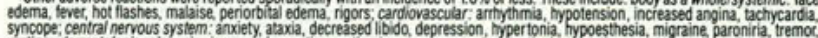

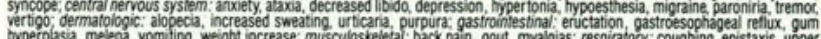

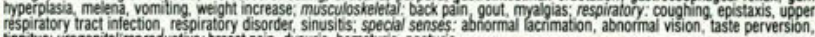
Adverse experiences which occurred in less than 1 in 1000 patients cannot be distinguished trom concurrent disease states or In multiple-dose U.S. and foreign controlled studies with nifedipine capsules in which adverse reactions were reported spontaneousk.
adverse ffects were frequent but generally not serious and rarely required discontinuation of theragy or dosage ad ustment. Most were ness. lightheadedness, and giddiness (27\%, compared to $15 \%$ placebo incidence): fiushing, hear sensation $25 \%$, compared to $8 \%$ placebo incidence); headache (23\%, compared to $20 \%$ placebo incidence); weakness $(12 \%$, compared to $10 \%$ placebo incidence)

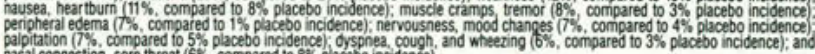

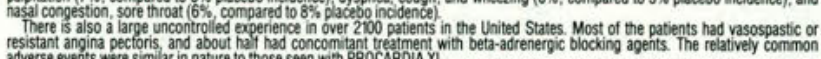
resistant angina pectoris, and about harse
adverse events were similar in nature to those seen with PROCARDIAXL

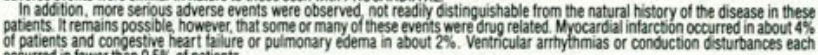

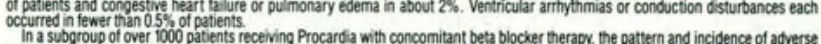

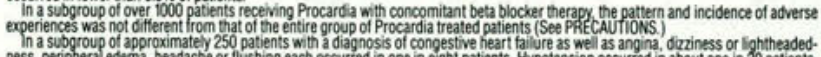
In a subgroup of approximatey 250 patients with a diagnosis of congestive feart fallure as well as angina, dizziness or lightheaded.
ness, peripheral edema, headache or flushing each occurred in one in eight patients. Hypotension occurred in about one in 20 patients.

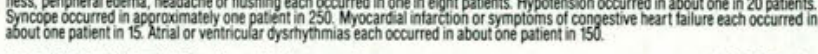
More detailed professional information avaliable on request.

o 1989, Pfizer Inc.

\section{Pfizer laboratories division \\ PFIZER INC.}

